DOI: $10.17816 /$ OV1093-96

\title{
ЛИМФАНГИОМА ОРБИТЫ
}

\author{
() В.В. Потёмкин ${ }^{1,2}$, О.А. Марченко ${ }^{1}$, Е.В. Агеева ${ }^{2}$ Ю.И. Малахова ${ }^{2}$ \\ ${ }^{1}$ СПбГБУЗ «Городская многопрофильная больница № 2 », Санкт-Петербург; \\ ${ }^{2}$ ФГБОУ ВО «Первый Санкт-Петербургский государственный медицинский университет \\ имени академика И.П. Павлова» Минздрава России, Санкт-Петербург
}

Для цитирования: Офтальмологические ведомости. - 2017. - Т. 10. - № 1. - С. 93-96

Дата поступления: 05.12.2016

Статья принята к печати: 20.02.2017

$\diamond$ В статье освещены клинические проявления, методы диагностики и варианты лечения лимфангиомы орбиты.

\& Ключевые слова: лифангиома орбиты.

\section{ORBITAL LYMPHANGIOMA}

\section{(C) V.V.Potemkin ${ }^{1,2}$, O.A. Marchenko ${ }^{1}$, E.V. Ageeva ${ }^{2}$, Yu.I. Malakhova ${ }^{2}$}

${ }^{1}$ Saint Petersburg State Hospital No 2, Saint Petersburg, Russian Federation;

${ }^{2}$ FSBEI HE Academician I.P. Pavlov First St Petersburg State Medical University of the Ministry of Healthcare of the Russian Federation, Saint Petersburg, Russia

For citation: Ophthalmology Journal, 2017;10(1):93-96

Received: 05.12 .2016

Accepted: 20.02 .2017

$\diamond$ In this article, clinical features, methods of diagnostic and treatment options of orbital lymphangioma are described.

^ Keywords: orbital lymphangioma.

\section{ЛИМФАНГИОМА ОРБИТЫ}

Лимфангиомы составляют от 1 до 3 \% всех опухолей орбиты. Диагностируются, как правило, у детей до 10 лет. Могут быть как изолированными, так и множественными, поражать одну или несколько областей одновременно. Для лимфангиом характерно длительное доброкачественное течение. Отличительной их особенностью является склонность к резкому увеличению размеров за счёт спонтанных кровотечений $[1,6,13]$.

Термин «лимфангиома» не является достаточно точным, поскольку подразумевает наличие новообразованных лимфатических сосудов

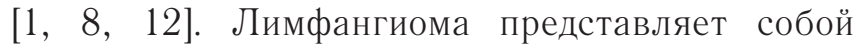
разрастание гетеротопических периваскулярных лимфатических сосудов. Состоит она из увеличенных, неинкапсулированных каналов, выстланных эндотелиальными клетками. В лимфангиоме нередко обнаруживают сосудистые компоненты, в том числе напоминающие вены. Наличие последних, по мнению нескольких авторов, обусловлено сочетанием лимфангиомы с артериовенозными пороками развития. Кровоизлияния из сосудов приводят к резкой прогрессии заболевания и формированию так называемых шоколадных кист - растянутых и заполненных кровью полостей $[1,8,16]$.

Согласно данным The International Orbital Society, все сосудистые мальформации можно разделить на три основные группы в зависимости от их гемодинамических взаимоотношений: 1) не связанные с системным кровотоком, 2) мальформации венозного кровотока, 3) мальформации артериального кровотока. Лимфангиомы, или так называемые лимфатические мальформации, относят к первой группе ввиду отсутствия связи с системным кровообращением [8].

Опухоль может располагаться как поверхностно, так и глубоко. По размерам различают 
макро- (>1 см) и микрокистозные $(<1$ см $)$ поражения $[1,4]$. В большинстве случаев лимфангиома проявляется медленно прогрессирующим экзофтальмом, птозом верхнего века, смещением и ограничением подвижности глазного яблока. В одном из исследований среди 26 пациентов с лимфатической мальформацией орбиты экзофтальм наблюдался у $85 \%$, птоз - у 73 \%, ограничение подвижности глазного яблока у $46 \%$ пациентов. Спонтанные кровоизлияния - наиболее опасное проявление лимфангиомы, которое может привести к экхимозу век, гипосфагме, резко выраженному экзофтальму и, как следствие, к резкому увеличению давления в орбите. Последнее является причиной развития компрессионной нейрооптикопатии и снижения зрения. Риск развития спонтанных кровоизлияний возрастает при инфекциях верхних дыхательных путей и травмах $[1,3,15,16]$.

Для данного вида мальформации характерен инфильтративный рост. Лимфангиома состоит из множества кист в виде «гроздьев винограда». В отличие от кавернозной гемангиомы, которая инкапсулирована и имеет чёткие границы, лимфангиома лишена чётких границ.

Для правильной постановки диагноза и для определения границ поражения необходимо применение дополнительных визуализирующих методов: ультразвукового исследования (УЗИ), компьютерной томографии (KT) и магнитно-резонансной томографии (МРТ).

На КТ лимфангиома имеет вид поликистозного образования, без чётких границ. МРТ лучше демонстрирует содержание кист. Типичными признаками при УЗИ являются неправильная форма с плохо очерченными границами, высокая рефлективность и отсутствие связи с сосудами $[2,5$, 9, 10].

Биопсия при лимфангиоме орбиты не является обязательной. Диагноз может быть поставлен на основании клинических проявлений и данных визуализирующих методов исследований $[1,2]$.

Можно выделить три основных варианта ведения пациентов с лимфангиомами: 1) наблюдение, 2) хирургическое иссечение, 3) альтернативные виды вмешательств.

Для лимфангиом в большинстве случаев характерно доброкачественное вялотекущее бессимптомное течение. При отсутствии угрозы снижения зрения эти пациенты требуют лишь наблюдения.

Показанием к началу лечения является снижение остроты зрения, которое обычно является следствием компрессионной нейрооптикопатии или экспозиционной кератопатии.

При хирургическом иссечении высок риск интра- и послеоперационных осложнений ввиду инфильтративного роста опухоли и отсутствия чётких границ с окружающими здоровыми тканями. Хирургическая тактика варьирует в зависимости от расположения, глубины поражения и степени вовлечения окружающих структур $[1,12]$.

Среди множества альтернативных видов лечения заслуживает внимания склерозирующая терапия. В качестве склерозирующих агентов применялись: блеомицин, доксициклин, пицибанил, тетрадецильный сульфат натрия и этанол. Эффективность применения каждого из этих агентов различна $[4,7,14,16]$.

В рамках данной статьи нам хотелось бы рассмотреть более подробно применение блеомицина в качестве склерозирующего агента. Ценность его в лечении лимфатических мальформаций была впервые проанализирована и представлена в 1977 г. японскими учёными во главе с исследователем Yra [17].

Блеомицин является цитотоксическим, противоопухолевым антибиотиком, разработанным Umezawa et al. в 1966 г. Он обладает специфичным склерозирующим эффектом на сосудистый эндотелий. В литературе имеются данные о применении блеомицина off label при врождённых сосудистых и лимфатических аномалиях головы, шеи и орбиты $[4,11]$. C. Gooding и D. Meyer сообщили о 4 успешных клинических случаях лечения лимфангиомы орбиты при помощи блеомицина [4].

Среди возможных побочных явлений можно выделить боль, красноту и отёк окружающих тканей. Упоминания о системных побочных эффектах такого вида терапии в литературе нет.

Вашему вниманию представлен клинический случай применения блеомицина при лимфангиоме правой орбиты.

В сентябре 2016 г. на 5-е микрохирургическое отделение СПб ГБУЗ «Городская многопрофильная больница № 2» по неотложной помощи был госпитализирован пациент 22 лет с жалобами на резкое снижение остроты зрения правого глаза, остро возникший экзофтальм, несмыкание глазной щели, выраженный отёк век справа. Со слов пациента, вышеописанные жалобы появились за день до поступления. Из анамнеза удалось выяснить, что больной с детства страдает лимфангиомой правой половины лица, но подобных эпизодов раньше не было. При поступлении острота зрения с коррекцией правого глаза составила 0,02 , 


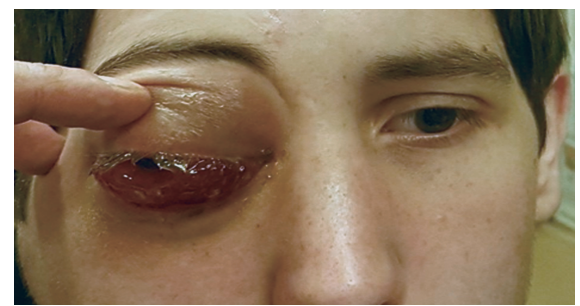

$a$

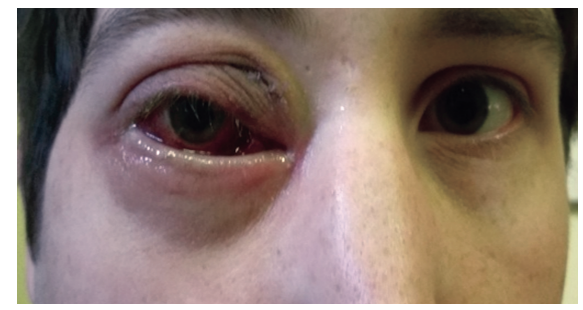

$b$

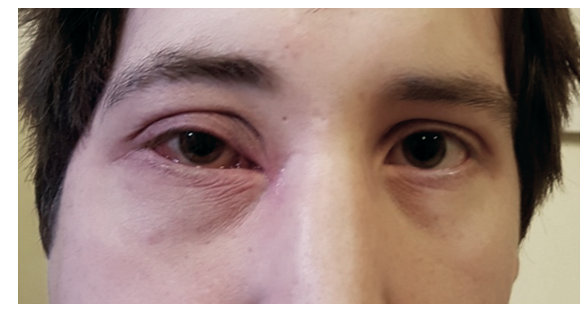

C

Рис. 1. Внешний вид пациента: $a$ - при поступлении, $b-$ через 10 дней после склеротерапии, $c$ - через 1 месяц после склеротерапии

Fig. 1. Appearance of a patient; $a$ - on admission, $b$ - in 10 days after sclerotherapy, $c$ - in 1 month after sclerotherapy

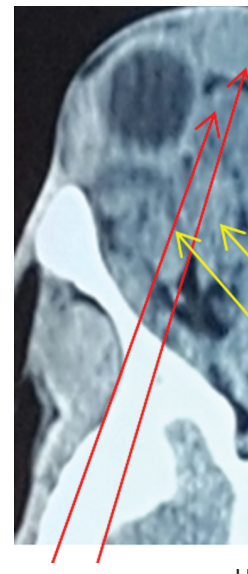

Гематические («шоколодные») кисты Gematic (chocolate) cysts

Новообразования во внутреннем хирургическом пространстве в виде «грозди винограда», сдавливающие зрительный нерв

The tumor in the inner surgical space shaped like a bunch of grapes, which compresses the optic nerve

Рис. 2. Компьютерная томограмма орбит

Fig. 2. Orbital CT scan левого глаза - 1,0. Объективно: умеренный отёк правой половины лица, резко выраженное выстояние правого глаза, лагофтальм, умеренный отёк век, офтальмоплегия, геморрагический хемоз справа, репозиция глазного яблока резко затруднена (рис. 1, a). При обследовании левого глаза патологических изменений выявлено не было. Пациенту была выполнена компьютерная томограмма орбит (рис. 2).

Учитывая высокий риск интраоперационных осложнений, было принято решение выполнить склерозирующую терапию блеоцином (блеомицина гидрохлорид, эквивалентный блеомицину 15 мг, Ниппон Кайяку КО, ЛТД, Япония) (рис. 3,b).

Процедура выполнялась под сочетанной внутривенной анестезией: через складку верхнего века была дренирована киста (рис. 3, a), получено 10 мл аспирационной жидкости (предполо-
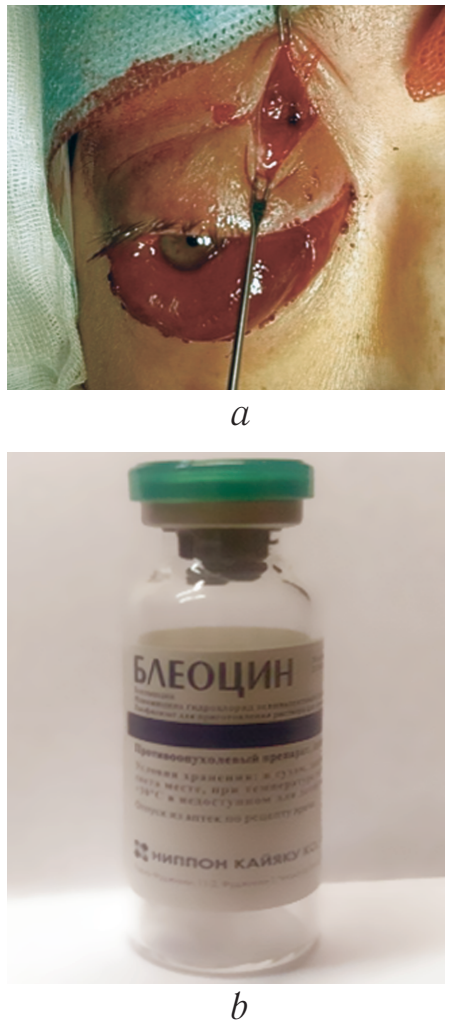
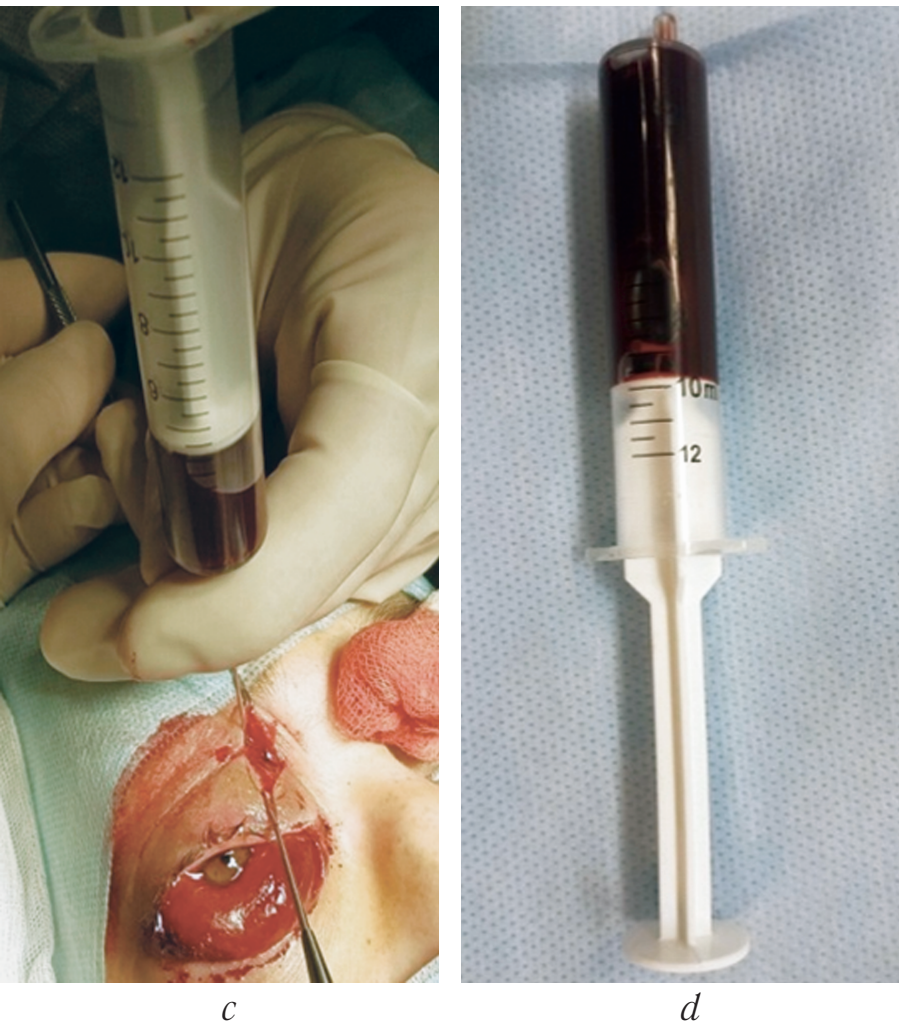

Рис. 3. Ход операции (описание в тексте)

Fig. 3. Surgical procedure (description in the text) 
жительно - крови) (рис. $3, c, d)$. Затем, сменив шприц, через ту же иглу было введено 10 ЕД блеоцина (из расчёта 1 мл блеоцина = 1 ЕД). В дополнение выполнена меридиональная конъюнктивотомия в косых меридианах. Кожа восстановлена узловыми швами (Vicryl 8-00).

Через неделю после процедуры острота зрения с коррекцией правого глаза повысилась до 0,2 , смыкание глазной щели было полным, экзофтальм значительно уменьшился. Через 1 месяц после процедуры острота зрения с коррекцией правого глаза составила 0,5. Эффект через неделю был обусловлен дренированием кисты, а через месяц склерозирующим эффектом блеомицина.

Единого подхода к лечению лимфангиом нет. Учитывая высокий рискпослеоперационныхосложнений, врач должен решить непростую задачу - выбрать тактику лечения. Склеротерапия зарекомендовала себя как эффективный и безопасный метод.

\section{СПИСОК ЛИТЕРАТУРЫ}

1. Бровкина А.Ф. Офтальмоонкология. Руководство для врачей.-М.:Медицина, 2002. [BrovkinaAF. Oftal'moonkologiya. Rukovodstvo dlya vrachey. Moscow: Meditsina; 2002. (In Russ.)]

2. Blaniuk LT. Orbital vascular lesions. Role of imaging. Radiol Clin North Am. 1999;37:169-183. doi: 10.1016/S00338389(05)70085-3.

3. Dryden RM, Wulc AE, Day D. Eyelid ecchymosis and proptosis in Iymphangioma. Am J Ophthalmol. 1985;100:486-487. doi: 10.1016/0002-9394(85)90526-4.

4. Gooding C, Meyer D. Intralesional bleomycin: a potential treatment for refractory orbital lymphangiomas. Ophthal Plast Reconstr Surg. 2014 May-Jun;30(3): e65-7.

5. Graeb DA, Rootman J, Robertson WD, et al. Orbital lymphangiomas: clinical, radiologic, and pathologic characteristics. Radiology. 1990May;175(2):417-21. doi: 10.1148/radiology.175.2.2326469.

Сведения об авторах

Виталий Витальевич Потёмкин - канд. мед. наук, доцент кафедры офтальмологии. ФГБОУ ВО «ПСПбГМУ им. И.П. Павлова» Минздрава России. Врач-офтальмолог. СПб ГБУЗ «Городская многопрофильная больница № 2», Санкт-Петербург. E-mail: potem@inbox.ru.

Ольга Анатольевна Марченко - врач-офтальмолог. СПб ГБУЗ «Городская многопрофильная больница № 2», СанктПетербург. E-mail: oamarchenko@yandex.ru.

Елена Владимировна Агеева - клинический ординатор, кафедра офтальмологии. ФГБОУ ВО «ПСПбГМУ им. И.П. Павлова» Минздрава России, Санкт-Петербург. E-mail: ageeva_elena@inbox.ru.

Юлия Ивановна Малахова - клинический ординатор, кафедра офтальмологии. ФГБОУ ВО «ПСПбГМУ им. И.П. Павлова» Минздрава России, Санкт-Петербург. E-mail: julia_ lady_m@mail.ru.
6. Henderson JW, Campbell RJ, Farrow GM, Garrity JA. OrbitalTumors. New York: Raven Press; 1994. 43-52 pp.

7. Hill RH, Shiels WE, Foster JA, et al. Percutaneous drainage and ablation as first line therapy for macrocystic and microcystic orbital lymphatic malformations. Ophthal Plast Reconstr Surg. 2012 Mar-Apr;28(2):119-25. doi: 10.1097//OP.0b013e318242ab0f.

8. Harris GJ. Orbital vascular malformations: a consensus statement on terminology and its clinical implications. Orbital Society. Am J Ophthalmol. 1999Apr;127(4):453-5.

9. Khan SN, Sepahdari AR. Orbital masses: CT and MRI of common vascular lesions, benign tumors, and malignancies. Saudi J Ophthalmol. 20120ct;26(4):373-83. doi: 10.1016/j.sjopt.2012.08.001.

10. Ko F, DiBernardo CW, Oak J, et al. Confirmation of and differentiation among primary vascular lesions using ultrasonography. Ophthal Plast Reconstr Surg. 2011Nov-Dec;27(6):431-5. doi: 10.1097/IOP.0b013e31822323af.

11. Paramasivam S, Fay A, Fifi J, Berenstein A. 0-015 image guided bleomycin sclerotherapy for orbital lymphatic malformation. J Neurointerv Surg. 2014Jul;6(Suppl):1:A8-9. doi: 10.1136/neurintsurg-2014-011343.15.

12. Saha $K$, Leatherbarrow $B$. Orbital lymphangiomas: a review of management strategies. Curr Opin Ophthalmol. 2012Sep;23(5):433-8. doi: 10.1097/ICU.0b013e3283560a99.

13. Shields JA, Bakewell B, Augsburger JJ, Flanagan JC. Classificationand incidence of space-occupying lesions of the orbit. Arch Ophthalmol. 1984;102:1606-1611. doi: 10.1001/archopht.1984.01040031296011.

14. Suzuki Y, Obana A, Gohto Y. Management of orbital lymphangioma using intralesional injection of OK-432. Br J Ophthalmol. 2000Jun;84(6):614-7. doi: 10.1136/bjo.84.6.614.

15. Shields JA. Diagnosis and management of orbital tumors. Philadelphia: WB Saunders; 1989. 149-169 p.

16. Tunç M, Sadri E, Char DH. Orbital lymphangioma: an analysis of 26 cases. Br J Ophthalmol. 1999;83:76-80. doi: 10.1136/bjo.83.1.76.

17. Yura J, Hashimoto T, Tsuruga N, Shibata K. Bleomycin treatment for cystic hygroma in children. Nippon Geka Hokan. 1977;46:607-14.

Information about the authors

Vitaly V. Potemkin - PhD, assistant professor. Department of Ophthalmology of the FSBEI HE Academician I.P. Pavlov First St Petersburg State Medical University of the Ministry of Healthcare of the Russian Federation, Saint Petersburg, Russia. Ophthalmologist. City Ophthalmologic Center of Saint Petersburg State Hospital No 2, Saint Petersburg, Russia. E-mail: potem@inbox.ru.

Olga A. Marchenko - Ophthalmologist. City Ophthalmologic Center of Saint Petersburg State Hospital No 2, Saint Petersburg, Russia. E-mail: oamarchenko@yandex.ru.

Elena V. Ageeva - resident. Department of Ophthalmology of the FSBEI HE "Academician I.P. Pavlov First St Petersburg State Medical University" of the Ministry of Healthcare of Russia, Saint Petersburg, Russia. E-mail: ageeva_elena@inbox.ru.

Yuliya I. Malakhova - resident. Department of Ophthalmology of the FSBEI HE "Academician I.P. Pavlov First St Petersburg State Medical University" of the Ministry of Healthcare of Russia, Saint Petersburg, Russia. E-mail: julia_lady_m@mail.ru. 